\title{
Renal manifestations of HIV during the antiretroviral era in South Africa: a systematic scoping review
}

\author{
Shirelle Assaram ${ }^{1 *}$, Nombulelo P. Magula1', Suman Mewa Kinoo² and Tivani P. Mashamba-Thompson ${ }^{3}$
}

\begin{abstract}
Background: It is estimated that 650,000 patients may develop human immunodeficiency virus (HIV)-related renal disease in South Africa. South Africa has recently adopted WHO policy, stipulating that all HIV-infected patients have access to antiretroviral treatment (ART) irrespective of CD4 cell count.

Methods: We searched Google Scholar, PubMed, Medline, Cochrane Library, Worldcat.org and EBSCO host databases from July 2015 to December 2015. Eligibility criteria included articles pertaining to renal manifestations of HIV in South Africa from 2004 to 2015 in adult patients ( $\geq 18$ years). We independently reviewed the articles for quality. Thematic content analysis was performed to identify patterns of renal manifestations from the included studies. The risk of bias (e.g. internal validity) in the included studies was evaluated using the mixed methods appraisal tool.

Results: Eleven out 21 studies were eligible for data extraction. The prevalence of urine abnormalities on urine dipsticks was high but had poor sensitivity and specificity for detecting renal impairment. Normal renal function occurred in 28.4 to $79 \%$ of patients, mild renal impairment occurred in 19 to $57.1 \%$ and moderate renal impairment in 2 to $14.4 \%$. Severe renal impairment occurred in $1.3 \%$ of patients. Both the Cockcroft-Gault equation (after correcting for bias) and the 4-variable Modification of Diet in Renal Disease equation (without the ethnicity factor for African Americans) have been validated for the estimation of glomerular filtration rate (eGFR) in Black South Africans. HIV-associated nephropathy was the most prevalent histology seen (57.2\%). Older age, a lower CD4 count, a low haemoglobin and a detectable viral load were associated with renal impairment. Renal function improved in the first year of commencing ART as evidenced by the regression of proteinuria and the increase in eGFR.
\end{abstract}

Conclusion: The findings of the review have implications to the recently adopted 'test and treat' approach to HIV prevention and management.

Systematic review registration: PROSPERO CRD42016039270

Keywords: HIV, Antiretroviral treatment, Renal failure, South Africa

\footnotetext{
* Correspondence: shirelleassaram@gmail.com

${ }^{1}$ Department of Internal Medicine, Nelson R. Mandela School of Medicine,

University of KwaZulu-Natal, 719 Umbilo Road, Congella, Durban 4013, South

Africa

Full list of author information is available at the end of the article
} 


\section{Background}

In April 2004, the South African National antiretroviral treatment (ART) guidelines were first implemented [1,2] and ART became universally accessible in the public sector for the first time. South Africa recently (01 September 2016) adopted the World Health Organization (WHO) "test and treat" approach, which was introduced as a possible means of controlling the global HIV epidemic [3]. This approach entitles every patient who tests positive for HIV to a lifelong ART regardless of their CD4 count or clinical staging [3]. With the successful rollout of ART in South Africa, the lifespan of people living with human immunodeficiency virus (HIV) has been prolonged thereby transforming HIV into a disease of chronicity [4], adding to the burden of infectious and non-communicable diseases [5]. Renal disease is a recognised complication of HIV infection and its incidence can be perpetuated by drug induced toxicity, comorbid diseases such as diabetes and hypertension and infectious diseases [6]. Data from the USA suggest that at some stage of their HIV infection, $10 \%$ of patients will develop HIVrelated renal disease [7]. If this is extrapolated to the South African context, it is estimated that 650,000 patients may develop HIV-related renal disease [7]. This large burden of chronic kidney disease (CKD) would place immense pressure on our resource-strained health system where access to renal biopsy, renal replacement therapies and nephrologists is limited [8].

Mayosi et al. report a $67 \%$ increase in deaths related to nephritis/nephrosis in South Africa from 1999 to 2006 causally linked to the increasing HIV prevalence [5]. In their systematic analysis, Stanifer et al. found that CKD is a prevalent and potentially growing disease in SubSaharan Africa with $24 \%$ of hypertensives, $18.9 \%$ of diabetics and $10 \%$ of HIV-infected patients having comorbid CKD [9]. The guidelines published by the Infectious Diseases Society of America (IDSA) in 2014 recommend that all individuals be assessed for kidney disease at the time of HIV diagnosis by way of a screening urinalysis for proteinuria and a calculated estimate of renal function in order to detect renal disease early [10]. The South African ART guidelines incorporate this policy, and the recent implementation of ART initiation irrespective of CD4 allows for earlier access to ART before the onset of advanced disease [3].

Despite the earlier initiation of ART and the screening for urinary abnormalities, renal disease in HIV infected patients is still prevalent [11]. In addition, HIV-associated nephropathy (HIVAN) has become the third leading cause of end stage renal disease among HIV infected AfricanAmerican patients $[12,13]$. To the best of our knowledge, this is the first systematic review that attempts to map the evidence of renal disease in people infected with HIV in South Africa post 2003 when ART became universally accessible to patients in the public sector. In light of the current upsurge of research and publications on the topic $[12,14-17]$, the contribution of a systematic scoping review gains importance and relevance by demonstrating the current data in order to identify research gaps and suggest novel ideas for future research.

\section{Methods}

\section{Study design}

The protocol of this study is registered in PROSPERO with registration number: CRD42016039270 and available via this website: http://www.crd.york.ac.uk/PROSPERO/ display_record.asp?ID=CRD42016039270.

In this study, we chose to undertake a systematic scoping review of published reviews as the best method to map the renal manifestations of HIV in South Africa during the ART rollout period from 2004 onwards. Guided by Arksey and O'Malley's scoping review framework [18], we searched cross-sectional studies, randomised controlled trials, non-randomised controlled trials, observational studies, review articles, case reports and systematic reviews that examined renal manifestations on HIV-infected patients in South Africa.

\section{Literature search}

We conducted a systematic literature search in the following databases: Google Scholar, PubMed, Medline, Cochrane Library, Worldcat.org and EBSCO host, for articles pertaining to renal manifestations of HIV in South Africa. The database search occurred from July 2015 to December 2015. The primary search terms related to HIV and the kidney specifically (i.e. HIV, kidney, renal, nephrology). The secondary terms were manifestations, renal failure, complications, South Africa, antiretroviral treatment, proteinuria and glomerular filtration rate (for a detailed description of the database search strategy, see Additional file 1). In order to direct the search to our research question, we used the filtering method which included the data range (2004 to 2015), human subjects, English language and adult patients ( $\geq 18$ years old). Medical subject headings $(\mathrm{MeSH})$ terms were also used.

\section{Eligibility criteria \\ Inclusion criteria}

- Evidence of renal manifestations in HIV infected patients in South Africa

- Evidence from the period 2004 to 2015 (ART rollout began in April 2004 in SA)

- Evidence of renal manifestations in adult HIV infected patients ( $\geq 18$ years old)

- English language publications

- Studies included irrespective of ART status, i.e. on treatment or not on treatment 


\section{Exclusion criteria}

- Evidence of renal manifestations in HIV infected patients outside South Africa

- Studies reporting exclusively on other HIV manifestations

- Studies before the ART era (prior to 2004 ART was not accessible in the public sector)

- Non English language studies

Study selection occurred in two stages. First, a single reviewer went through the titles from the database search and decided on eligibility based on the inclusion and exclusion criteria. For example, titles stating research carried out in an ineligible country would be excluded. If a reviewer was uncertain of the eligibility of a title, it was not excluded but rather carried onto the next stage of the selection process. In the second stage, two independent reviews of the titles and abstracts, using inclusion and exclusion criteria, was undertaken. Discrepancies were resolved by discussion until consensus was reached. The remaining articles were then assessed for eligibility for data extraction. A PRISMA flow diagram (Fig. 1: Literature Search and Selection of Studies) shows the process involved in obtaining eligible studies. The PRISMA checklist is provided as an Additional file 2.

\section{Quality of the evidence}

The risk of bias (overall quality of each article) in the included studies was evaluated using the mixed methods appraisal tool (MMAT) for mixed methods studies [19] (Additional file 3). The risk of bias scale scored studies was based on 14 criteria. Quantitative studies were assessed by the following domains: the clarity of the research questions, confidence in the assessment of the research question, appropriateness of data sources collected, suitability of statistical analysis to address the research question, confidence in the assessment of exposure, acknowledgement of possible researcher bias, appropriateness of the sampling strategy, representativeness of population, confidence in the measurements outcome and an acceptable response rate. The nonrandomised study was assessed by the following domains: minimization of selection bias during recruitment, confidence in the assessment of exposure, matching exposed and unexposed variables for all those that are associated with the outcome of interest or the adjustment of statistical analysis for these prognostic factors, confidence in the assessment of outcome or adequacy of the follow-up of cohorts. For each included study, an overall percentage quality score was calculated. The scores are presented using descriptors such as *( $\leq 25 \%)$-poor, $* *(26-50 \%)$-fair, ***** $(51-75 \%)$-average, and ***** (76-100\%)-good (Additional file 3). Two reviewers (SA and SMK) independently performed each quality assessment. Differences in ratings were resolved through discussion.

\section{Thematic analysis}

Thematic content analysis was performed to identify patterns of renal manifestations from the included studies. The included manuscripts were manually coded into categories which were grouped into the following five themes:

- Urine analysis

- Estimated glomerular filtration rate

- Renal biopsy

- Risk factors for renal dysfunction

- Clinical and histological responses to ART

\section{Results}

A total of 6808 articles were retained from our initial search. Applying our exclusion criteria reduced the number of studies to 11 (Figure 1). Level of agreement between reviewers was 80 versus $50 \%$ expected by chance. This constitutes moderate to substantial agreement, with estimated kappa statistic $=0.2295 \%$ (confidence interval $[\mathrm{CI}]$ $0.70 ; 0.26)$.

A total of 6758 studies were excluded as they did not meet the inclusion criteria for this study. Of those, 10 underwent a full manuscript review and were found to have no valuable data for analysis in this study for the following reasons: not within the specified time frame $[12,17,20-23]$, outcomes of chronic haemodialysis [24], systematic review and meta-analyses of countries in Sub-Saharan Africa (9), irrelevant study population [25], and a dissertation published at a later stage [26].

\section{Characteristics of included studies}

Eleven out of the 21 reviewed articles were eligible for data extraction (Additional file 4). Of these, eight were conducted in an urban setting [15, 16, 27-32] and three were carried out in a rural setting [33-35]. Rural is defined as sparsely populated areas in which people farm or depend on natural resources, including the villages and small towns that are dispersed through these areas. In addition, in South Africa, they include the large settlements in the former homelands, created by the apartheid removals, which depend for their survival on migratory labour and remittance. All included studies were published between 2008 and 2015. Study participants were predominantly female (total female percentage $57.9 \%$ ) in all included studies except two [29, 30]. The total sample size of all 11 studies was 6595 participants. The included number of participants in each study was 100 participants or more. The average age in all the included studies was $38.6 \pm 4.5$ years. The study designs of the included studies were as follows: two 


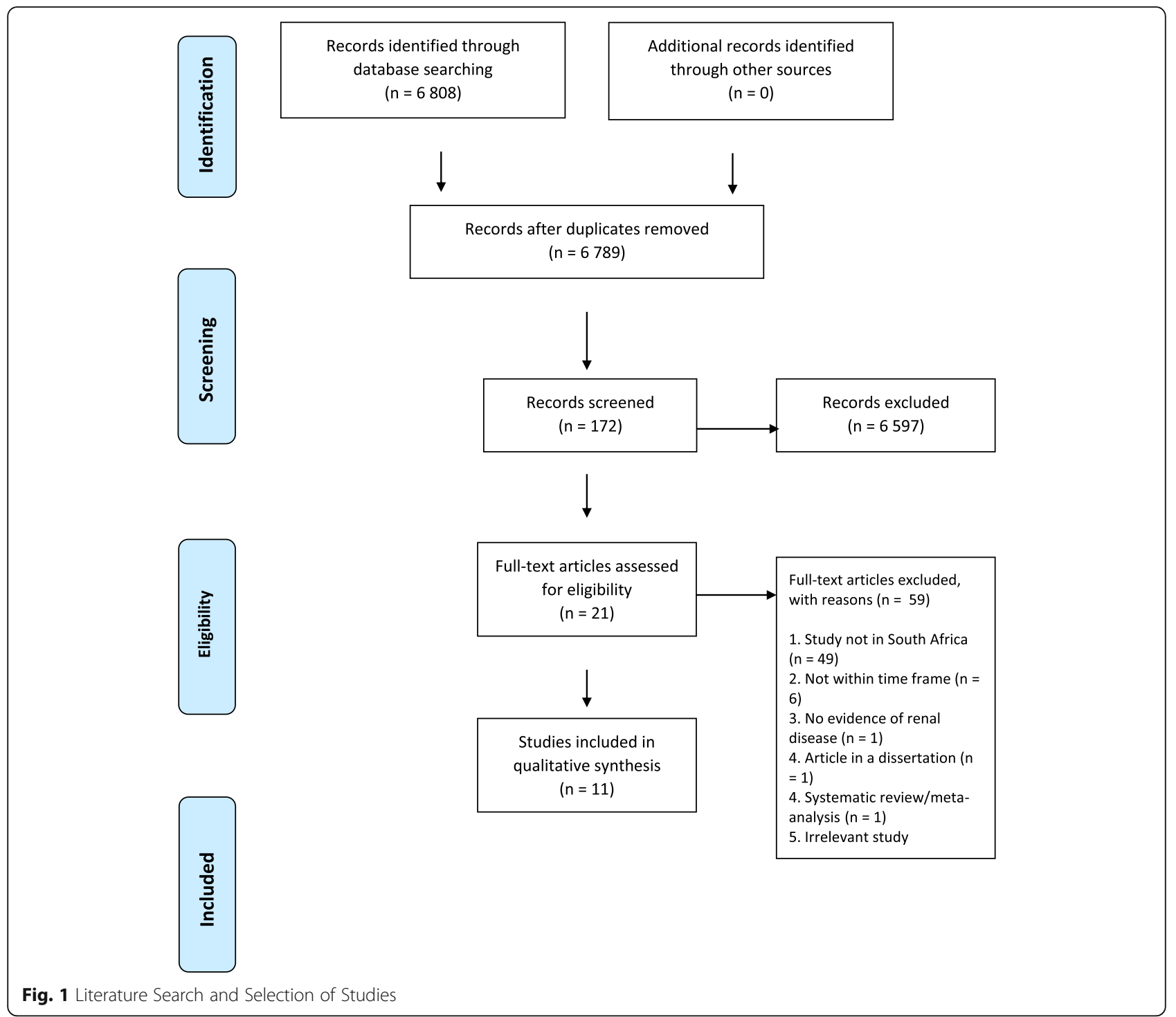

retrospective cohort studies [27, 29], one cohort study [31], two prospective cohort studies [32, 33], one nonrandomised control study [15], one case control study [28], and four cross-sectional studies [16, 30, 34, 35].

All the included studies were aimed at assessing the renal status, risk factors and renal outcomes in HIVinfected patients either before ART, whilst on ART or both. Seven of the 11 studies (63.6\%) assessed for the prevalence of renal impairment in HIV-infected patients $[16,27,29,32,33,35]$, two studies $(18.1 \%)$ analysed renal biopsies to evaluate the clinical and histological responses of HIV-associated kidney disease to ART $[15,31]$, one study (9\%) assessed the performance of the 4-variable Modification of Diet in Renal Disease (4-v MDRD) and Cockcroft-Gault (CG) equations for estimating glomerular filtration rate (eGFR)/creatinine clearance in South African Black patients [30], and one study (9\%) extracted genomic DNA from kidney samples to determine the prevalence of APOL1 risk variants and their effect on CKD in Black South Africans [28]. General and specific characteristics of all included studies are described in Additional file 4. The evidence of renal dysfunction in the included studies is summarised in Table 1.

\section{Risk of bias assessment}

The studies' quality scores ranged from 87.5 to $100 \%$ (Additional file 2). Six of the 11 included studies scored the highest quality score of $100 \%[15,28,29,31-33]$. The three studies scoring the lowest had an average score of $87.5 \%$ each [30,34], and the remaining two studies had an average score of $91.5 \%$ each [16, 35]. Overall, the body of evidence was considered at minimal risk of bias due to the following: description of 
Table 1 Evidence of renal dysfunction in the included studies

\begin{tabular}{|c|c|c|}
\hline Author & Markers for renal impairment & Definition of renal disease \\
\hline Brennan et al. 2011 [27] & $\begin{array}{l}\text { Creatinine clearance using } \\
\text { Cockcroft-Gault (CG) equation. }\end{array}$ & $\begin{array}{l}\text { Nephrotoxicity defined as any decline in } \\
\text { kidney function from baseline (acute or } \\
\text { chronic) that is secondary to a toxin } \\
\text { (including drugs) documented within } \\
48 \text { months of initiating tenofovir (TDF). } \\
\text { Normal renal function ( }>90 \mathrm{ml} / \mathrm{min} \text { ), } \\
\text { mild renal dysfunction ( } 60-89 \mathrm{ml} / \mathrm{min} \text { ) } \\
\text { and moderate renal dysfunction } \\
\text { (30-59 } \mathrm{ml} / \mathrm{min} \text { ) }\end{array}$ \\
\hline
\end{tabular}

Fabian et al. 2009 [16]

Urine dipstick for proteinuria and microalbuminuria

Fabian et al. 2013 [15]

Franey et al. 2009 [33]

Kamkuemah et al. 2015 [32] eGFR calculated using CG

Madala et al. 2014 [34]

Vachiat et al. 2013 [29]

Wearne et al. 2012 [31] equation

Urine dipstick for proteinuria. Estimated glomerular filtration rate (eGFR) using both CG and 4 variable Modification of Diet in Renal Disease (MDRD) formulae. Renal biopsy

Urine dipstick for proteinuria. eGFR using the four variable MDRD equation. Urine dipstick for proteinuria

Urine dipstick for proteinuria. eGFR calculated using the MDRD equation for $\geq 18$ years and the Schwartz equation for $<18$ years old.

rine for proteinuria either dipstick or spot urine protein creatinine ratio.

Serum creatinine levels.

Renal biopsy

Serum creatinine

Urine protein creatinine ratio
Results

The risk of nephrotoxicity and death by

48 months increased with decreasing renal function at initiation of TDF. Patients switched onto TDF had a higher risk of nephrotoxicity and death compared to ART naïve patients. Median time to nephrotoxicity after TDF initiation is 3.6 months confirming the importance of the month 3 creatinine clearance.

$18.5 \%$ had microalbuminuria, $6.4 \%$ had overt proteinuria and $2.4 \%$ had nephrotic range proteinuria. independent of sex; overt proteinuria: protein-to-creatinine ratio of $0.03-0.3 \mathrm{~g} / \mathrm{mmol}$; nephritic range proteinuria: protein-tocreatinine ratio $>0.3 \mathrm{~g} / \mathrm{mmol}$.

Persistent microalbuminuria: microalbumin-to-creatinine ratio of 3.4$33.9 \mathrm{mg} / \mathrm{mmo}$

Persistent overt proteinuria: protein-tocreatinine ratio of $34 \mathrm{mg} / \mathrm{mmol}-0.3 \mathrm{~g} /$ mmol;

Nephrotic proteinuria: protein-tocreatinine ratio $>0.3 \mathrm{~g} / \mathrm{mmol}$.

Severe renal impairment: eGFR < 30 mls/ $\min / 1.73 \mathrm{~m}^{2}$;

Moderate renal impairment: eGFR 30

$60 \mathrm{mls} / \mathrm{min} / 1.73^{2}$.

mild renal impairment: eGFR 60-90 m/s/ $\min / 1.73^{2}$.

Proteinuria $\geq 1+$ protein on dipstick Renal dysfunction defined as either reduced eGFR and/or proteinuria/ haematuria.

Severe renal function reduction was defined as eGFR $<30 \mathrm{ml} / \mathrm{min} / 1.73 \mathrm{~m}^{2}$, moderate reduction as eGFR of 30 $59 \mathrm{ml} / \mathrm{min} / 1.73 \mathrm{~m}^{2}$ and mild reduction as an eGFR of $60-89 \mathrm{ml} / \mathrm{min} / 1.73 \mathrm{~m}^{2}$.

Chronic kidney disease (CKD) defined by eGFR $<60 \mathrm{ml} / \mathrm{min} / 1.73 \mathrm{~m}^{2}$ and/or proteinuria and/or abnormal renal ultrasound, persistent for $\geq 3$ months.

Acute kidney injury (AKI) defined as an improvement in admission serum creatinine of $>50 \%$. They were further subdivided using the rifle criteria: risk—serum creatinine < $194 \mu \mathrm{mol} / \mathrm{L}$; injury-serum creatinine 195 to $291 \mu \mathrm{mol} / \mathrm{L}$; and failure-serum creatinine > $291 \mu \mathrm{mol} / \mathrm{L}$

HIV-associated nephropathy (HIVAN) defined as a constellation of glomerular interstitial and tubular abnormalities, and there must be epithelial cell hyperplasia if only tubular or interstitial disease was present.
9\% had normal renal function at baseline, 19\% had mildly reduced renal function and $2 \%$ had moderate rena impairment at baseline. Overall renal function improved over the first year after starting TDF-containing ART regimens.

$68 \%$ had microalbuminuria, 23\% had overt proteinuria and $9 \%$ had nephrotic proteinuria.

There was an improvement in eGFR on antiretroviral treatment (ART). There was proteinuria in response to treatment Despite the rapid clinical response to ART, there was relative lack of histological resolution.

Severe renal impairment was uncommon while moderate and mild renal impairment were more common. Mild and moderate renal impairment improve on ART. Urine analysis may not be sufficiently sensitive to be used as a single screening test for renal disease at baseline.

eGFR was $<30 \mathrm{ml} / \mathrm{min} / 1.73 \mathrm{~m}^{2}$ in $50.6 \%$ of patients as this was a CKD clinic. Main risk factors for CKD were diabetes, hypertension and HIV.

Majority had AKI 56\%, followed by CKD $23 \%$ and $21 \%$ had acute on chronic kidney disease. Proteinuria did not patients with AKI. AKI was common in HIV-infected patients and occurred at a younger age than HIV negative patients.

For patients with HIVAN, there was an improvement in proteinuria and stabilisation of renal function after commencing ART. Renal biopsy is essential to diagnose renal disease in HIV-infected patients. partial or complete remission of predict recovery or death in HIV-infected 
Table 1 Evidence of renal dysfunction in the included studies (Continued)

\begin{tabular}{|c|c|c|c|}
\hline Author & Markers for renal impairment & Definition of renal disease & Results \\
\hline Wensink et al. 2015 [35] & $\begin{array}{l}\text { Urine for albuminuria } \\
\text { eGFR calculated using MDRD and } \\
\text { CKD epidemiology collaboration } \\
\text { formula }\end{array}$ & $\begin{array}{l}\text { Moderately increased albuminuria: } \\
\text { albumin creatinine ratio } 30 \text { to } 299 \text { mg/g; } \\
\text { Severely increased albuminuria: albumin } \\
\text { creatinine ratio }>300 \mathrm{mg} / \mathrm{g} \text {. }\end{array}$ & $\begin{array}{l}\text { Albuminuria occurred in } 20 \% \text { of patients } \\
\text { while only } 2 \% \text { had eGFR }<60 \mathrm{ml} / \mathrm{min} / \\
1.73 \mathrm{~m}^{2} \text {. Higher eGFR was significantly } \\
\text { linked to lower prevalence of } \\
\text { albuminuria. Albuminuria was linked to } \\
\text { higher frequency of diabetes, } \\
\text { hypertension, high total cholesterol and } \\
\text { decreased eGFR. }\end{array}$ \\
\hline
\end{tabular}

CG Cockcroft-Gault, TDF tenofovir disoproxol fumarate, eGFR estimated glomerular filtration, MDRD Modification of Diet in Renal Disease, ART antiretroviral treatment, CKD chronic kidney disease, AKI acute kidney injury, HIV human immunodeficiency virus, HIVAN HIV-associated nephropathy

randomisation was clear, allocation concealment none, retention percentage in study was acceptable, minimal selection bias and, selection and comparativeness of the control groups was adequate.

\section{Findings of the study}

Kidney disease is a complication of HIV infection and can present as acute kidney injury (AKI) or CKD. Only one study assessed AKI (defined as an improvement in admission serum creatinine of $>50 \%$ ) in HIV-infected ART naïve patients [29]. Their findings demonstrated that $56 \%$ had AKI, 21\% had acute on chronic kidney disease and $23 \%$ had CKD [29]. The causes of AKI included sepsis, volume depletion, haemodynamic instability, toxins, urological obstruction and miscellaneous causes [29].

\section{Urine analysis}

The incidence of urine abnormalities on urine dipsticks was high: 30 to $57 \%$ had leukocyturia [16, 29], 16 to $40 \%$ had microscopic haematuria $[16,29,33]$ and 20 to $44 \%$ had microalbuminuria/proteinuria $[15,16,33,35]$. Two studies investigated the cause of leukocyturia and detected an infective organism, mainly E. coli, in $29.1 \%$ of culture positive cases in one study (ART naïve outpatients) [16] and in $8.9 \%$ of culture positive cases in the other study (ART naïve outpatients with renal failure) [29]. Franey et al. performed urine dipstick analysis on 149 patients who were initiating ART in a rural clinic, to assess its utility to detect impaired renal function [33]. They concluded that urine dipsticks analysis alone had poor sensitivity and specificity for detecting impaired renal function (PPV 0.22) [33].

In three studies on ART naïve patients, positive proteinuria on dipsticks was quantified by sending the specimen for a spot protein:creatinine ratio (PCR) $[15,16,29]$. If the dipsticks were negative for protein, they were screened for microalbuminuria which, if positive, were then sent for a spot microalbumin-tocreatinine ratio test (MCR) $[15,16]$. In their study, Fabian et al. found that, of the 253 dipsticks positive for proteinuria on ART naïve patients, only 193 were confirmed by the laboratory [16]. Wensink et al. [35] submitted random urine samples directly to the lab for the albumin:creatinine ratio (ACR), bypassing urine dipsticks analysis. Two studies quantified the degree of proteinuria using either the MCR [15] or the ACR [35]. Microalbuminuria (MCR $3.4-33.9 \mathrm{mg} / \mathrm{mmol}$ ) occurred in $68 \%$ of patients, overt proteinuria (MCR $34 \mathrm{mg} / \mathrm{mmol}-0.3 \mathrm{~g} / \mathrm{mmol}$ ) in $23 \%$ and nephrotic proteinuria ( $\mathrm{MCR}>0.3 \mathrm{~g} / \mathrm{mmol}$ ) in 9\% of ART naïve patients [15]. Similar figures were noted in the study that quantified the ACR in a mixed study population predominantly on ART ( $87 \%$ on ART): $20 \%$ of patients had moderately increased albuminuria (ACR 30$299 \mathrm{mg} / \mathrm{g}$ ) and $1 \%$ had severely increased albuminuria (ACR $>300 \mathrm{mg} / \mathrm{g}$ ) [35]. In the same study, a detectable HIV viral load, hypertension, total cholesterol and eGFR were all independently associated with albuminuria [35].

\section{Estimated glomerular filtration rate (eGFR)}

Six studies utilised eGFR as a marker of renal dysfunction $[15,27,30,32,33,35]$. eGFR was calculated using the CG equation [27, 32], the 4-v MDRD equation [33], comparing the CG and 4-v MDRD equations $[15,30]$ or comparing the 2009 Chronic Kidney Disease Epidemiology Collaboration (CKD-EPI) formula and the 4-v MDRD equation [35]. Both the CG equation (after correcting for bias) and the 4-v MDRD equation (without the ethnicity factor for African Americans) have been validated for the estimation of eGFR in Black South Africans [30].

The eGFR was classified as follows: severe renal impairment eGFR $<30 \mathrm{mls} / \mathrm{min} / 1.73 \mathrm{~m}^{2}$, moderate renal impairment eGFR $30-59 \mathrm{mls} / \mathrm{min} / 1.73 \mathrm{~m}^{2}$, mild renal impairment eGFR $60-89 \mathrm{mls} / \mathrm{min} / 1.73 \mathrm{~m}^{2}$ and normal renal function eGFR $>90 \mathrm{mls} / \mathrm{min} / 1.73 \mathrm{~m}^{2}$. Using the afore-mentioned classification, baseline (before ART initiation) renal function was documented as follows: normal renal function occurred in $28.4 \%$ to $79 \%$ of patients, mild renal impairment occurred in 19 to $57.1 \%$ and moderate renal impairment in 2 to $14.4 \%$ [27, 32, 33]. Baseline severe renal impairment occurred in $1.3 \%$ of ART naive patients in the study by Franey et al. [33]. Wensink et al. had a mixed study population of ART naïve patients and HIV-infected patients on ART and the majority of patients had normal renal function, $2 \%$ had moderate renal impairment and $10 \%$ had mild renal impairment [35]. 
Brennan et al. confirmed the importance of the 3month creatinine clearance by demonstrating that the median time to nephrotoxicity after tenofovir disoproxil fumarate (TDF) initiation was 3.6 months [27]. A study by Kamkuemah et al. showed that early serum creatinine testing at months 1 and 2 after initiation of TDF may not be useful in predicting those at risk for renal dysfunction as it had a low predictive value for predicting change in eGFR after a year on ART [32].

\section{Renal biopsy}

Renal histological findings have been inconsistently classified in patients on ART [31]. HIV-associated nephropathy (HIVAN) was the most prevalent histology seen (57.2\%) followed by HIVAN with immune complex glomerulonephritis (ICGN) at 21.8\%; without HIVAN or ICGN (12.5\%); and ICGN in isolation (8.3\%) [31]. However, in another study by Fabian et al., there was a paucity of HIVAN lesions on histology [15].

\section{Risk factors for renal dysfunction}

Two studies were unanimous that abnormal renal function at baseline was associated with older age, World Health Organizaton (WHO) stage III/IV disease and a lower CD4 count $[32,33]$. In addition to these variables, a low haemoglobin and a detectable viral load were also associated with abnormal renal function in a mixed cohort of ART naïve patients and patients on ART [27, 32]. In the same study, patients who were switched onto TDF from another regimen had a higher risk of nephrotoxicity and death, than ART naïve patients initiated on TDF [27]. Younger age and advanced immunosuppression were found to be risk factors for AKI [29]. There was no consensus regarding gender as a risk factor for renal dysfunction, as one study found women to be at greater risk [32] whilst another found men to be at greater risk [33]. The APOL1 risk allele found on chromosome 22 occurred in more than $30 \%$ of African American individuals with HIVAN [36]. Kasembeli et al. showed that $79 \%$ of Black patients with HIVAN carried two copies of APOL1 risk alleles as opposed to $2 \%$ in the general population, suggesting that ART naive Black South Africans were at high risk of developing HIVAN(28). CKD (defined as eGFR $<60 \mathrm{ml} / \mathrm{min} / 1.73 \mathrm{~m}^{2}$ and/or proteinuria and/or abnormal renal ultrasound persistent for $\geq 3$ months) risk factors which were noted in two studies of South African patients attending CKD clinics, were hypertension (36 to $77.8 \%$ ), diabetes (25 to $29.8 \%$ ) and HIV (20 to $28.5 \%$ ) [30]. It is interesting to note that $51.1 \%$ of these patients had more than one CKD risk factor [34].

\section{Clinical and histological response to ART}

In the majority of patients, renal function improved in the first year of commencing ART, as evidenced by the regression of proteinuria and the increase in eGFR [15, 31, 32]. However, there was no significant change in morphology noted on histology before and after ART initiation to explain the improvement in proteinuria [15].

\section{Discussion}

We conducted a systematic scoping review of the available literature on the renal manifestations of HIV in the era of ART rollout in South Africa from 2004. This review provided a general overview of renal impairment (diagnostics, histological features and risk factors) in South African HIV-infected patients mainly prior to ART initiation. Most of what we know about HIVrelated kidney disease has come from research performed in high income countries where the patient profiles and demographics are discordant to that of South Africa. The salient points unravelled by our review indicate a paucity of data on ART-related renal complications, specifically TDF nephrotoxicity; a deficiency of research on the impact of ART on AKI and CKD and the long-term outcomes of renal disease for patients on ART; and a relatively unknown prevalence of HIV-related kidney disease for patients on ART in South Africa. Bearing in mind that South Africa recently (1 September 2016) adopted the WHO "test and treat" approach to HIV prevention and management [3], these findings has major implications for the near future.

TDF is widely used as first line ART in South Africa since April 2010 as part of National ART guidelines [37]. A biopsy series revealed that TDF nephrotoxicity is essentially a reversible form of toxic acute tubular necrosis with features of mitochondrial injury [38]. Numerous studies have demonstrated a decrease in kidney function with TDF usage and suggest monitoring of renal function to prevent TDF nephrotoxicity [27, 39-41]. Early detection of proteinuria using accurate screening tests is important to decrease nephrotoxicity and improve outcomes in HIV-infected individuals [27]. The current South African ART guidelines recommend routinely performing urine dipstick analysis on patients before and while on ART, as well as assessing the serum creatinine and eGFR at baseline (prior to ART initiation) and then at 3 months, 6 months and annually thereafter for patients on TDF. The rate of compliance to these guidelines, however, is not known.

The use of urine dipsticks alone as a screening tool has become a contentious issue with research both locally [33] and overseas [42] suggesting the poor validity of urine dipsticks in detecting proteinuria. Data from another South African study, Han et al., suggested that 
HIVAN is possible in patients without overt nephrotic syndrome and in patients who have only microalbuminuria. Therefore, microalbuminuria can be considered an early marker of HIVAN [12]. Szczech et al. concluded that miroalbuminuria predicts the development of proteinuria in HIV-infected patients [43]. Stanifer et al. showed that the prevalence of proteinuria when measured in people with HIV, hypertension or diabetes is substantial. However, the best method of urine protein detection is unknown [9]. Currently, our urine dipstick analyses detect proteinuria only (not microalbuminuria). In other countries, alternate methods of urine analysis are already being sought. In a recent study set in Mexico City, a comparison was made between the protein reagent strip (PRS) and the urinary protein/creatinine ratio (uPCR) to detect proteinuria. It was concluded that there was a high concordance between the detection of urine protein by PRS and UPCR and therefore the PRS could be useful in low income countries to detect proteinuria [44].

Two South African studies in our review demonstrated the importance of an early creatinine clearance, prior to 4 months after the initiation of TDF [27, 32]. Both the CG equation (after correcting for bias) and the 4-v MDRD equation (without the ethnicity factor for African Americans) have been validated for the estimation of eGFR in Black South Africans [30]. The production of creatinine is determined mainly by muscle mass and dietary intake [45]. Therefore, using the ethnicity factor in the 4-v MDRD equation overestimates the eGFR in Black South Africans [30] due to differences in diet, muscle mass and body composition between Black South Africans and African Americans [30]. In the current review, the majority of the patients had mild to moderate renal dysfunction with a low prevalence of severe renal impairment prior to ART initiation. Similar findings were seen in a study by Overton et al. set in Washington [46]. The prevalence of significant renal impairment in a South African study [33] was slightly higher than that of a Kenyan study [47], possibly because of the differences in the two African populations and because the eGFR method used in the South African study has not been validated for use in other African countries. Though the prevalence of chronic renal failure is low in the HIV-infected population, it has been shown to be higher than that of the general population in an American study [46]. One South African study showed that approximately $6 \%$ of ART naïve outpatients who presented with proteinuria had HIV-related kidney disease [12] and another study of 99 in-patients who underwent renal biopsy showed that 27 patients (27.3\%) had HIVAN, only 3 of which were on ART [17].

In the current review, HIVAN was the most common histology seen on renal biopsy $[15,31]$. Other histologies have been documented in various studies both locally [12, 17, 22, 31] and overseas [48]. The third leading cause of end stage renal disease (ESRD) in African Americans aged between 20 and 64 years was found to be HIVAN [13]. HIVAN is commonly found in HIVinfected Black patients, suggesting a role of genetics in the development of HIVAN [13]. Kasembelli et al. proved that Black South Africans who carried two copies of the APOL1 risk alleles were at higher risk of developing HIVAN [28]. Lucas et al. demonstrated that ART can prevent or reduce the risk of developing HIVAN and, should it occur, patients on ART may have a slower course and lower mortality than patients not on ART [49]. Following the diagnosis of HIVAN, patients can progress to end stage renal failure within months [17]. In a South African study over a 10 year period of renal biopsies, the incidence of HIVAN increased from $6.6 \%$ in 2000 to $25.7 \%$ in 2009 [22]. Furthermore, of the 27 patients diagnosed with HIVAN on renal biopsy in a study in South Africa, only 3 were on ART [17]. This reaffirms the importance of renal biopsy in accurately diagnosing renal disease, particularly HIVAN [12, 17, 48], so that ART can be initiated early to improve outcomes. Sadly, due to a lack of resources and specialists, access to renal biopsy is limited to urban tertiary hospitals in South Africa.

From September 2016, when ART will have been made universally available to all HIV-infected South Africans in accordance with WHO ART guidelines [3], even patients with undiagnosed HIVAN will have been initiated on ART. We know from studies in other countries that renal function improves with ART. In a randomised ART trial in Uganda and Zimbabwe, there was stabilisation or a slight improvement in renal function after the initiation of ART [50]. Another study demonstrated the resolution of renal disease with ART and the recurrence of renal disease after stopping ART [51]. TDF-induced renal toxicity usually resolves after TDF cessation, but the TDF-related renal damage is not always completely reversible [52]. Our systematic scoping review demonstrated that renal function essentially improved on ART [15, 31, 32]. However, data on the long-term outcomes of renal function and CKD for patients on ART in South Africa is lacking. It was noted that 2015 had the most publications applicable to the topic of our scoping review, indicating a recent increase in interest on this subject in South Africa.

\section{Recommendations for future research}

The majority of the included studies were conducted in an urban setting where access to healthcare and laboratories is readily available. However, research shows the high HIV prevalence and fewer patients on ART, in rural South Africa [53]. Females bear the brunt of the HIV epidemic in South Africa having a significantly higher 
prevalence of HIV than their male counterparts [53]. In the current review, consensus was not reached regarding gender as a risk factor for renal dysfunction possibly because females predominated in most studies. Therefore, comparative studies to determine the differences in renal manifestations between HIV-infected males and females, preferably in rural settings, are recommended. We also need to recruit more males to participate in research.

Currently, our urine dipstick analyses detect only proteinuria and not microalbuminuria. This suggests a novel idea for research: looking at the significance of microalbuminuria versus proteinuria in our HIVinfected population, the outcome of which could guide future urine diagnostics in baseline screening for renal disease. Cost-effective methods for urine screening and estimating GFR are needed, especially for rural areas where access to laboratories is limited.

Considering that the majority of South African HIVinfected patients are Black and with a genetic predisposition to HIVAN, we anticipate that the prevalence of undiagnosed HIVAN will be significant. As far as we know, there are no prospective randomised controlled trials in South Africa investigating treatment options for HIVAN and no data on the actual prevalence of HIVAN.

Data on TDF nephrotoxicity and its impact on longterm renal function are lacking in South Africa. Prospective clinical trials focusing on TDF nephrotoxicity and its long-term renal outcomes are needed.

The overall prevalence of renal disease in South Africa is unknown. We urgently need epidemiological studies assessing the prevalence of renal disease and HIVrelated renal disease in South Africa in order to efficiently plan and sustain an effective CKD programme. The establishment of renal registries will assist with much needed statistics on the morbidity and mortality of renal disease in general and specifically to HIV and ART.

\section{Implications for practice}

Risk factors associated with proteinuria and albuminuria in HIV-infected patients (low eGFR, older age, diabetes and HPT) $[54,55]$ overlap with those for CKD patients [56]. Stanifer et al. found that $24 \%$ of hypertensives, $18.9 \%$ of diabetics and $10 \%$ of HIV-infected patients have co-morbid CKD in Sub-Saharan Africa [9]. With the high burden of HIV and non-communicable diseases such as CKD, hypertension and diabetes in South Africa, it would be wise for the Health Department to invest in CKD clinics in rural areas and nephrology outreach services as previously proposed by Madala et al. [34]. All staff must be trained in screening for renal dysfunction and referral pathways and support systems must be in place for patients to access specialist care. In South Africa, the number of nephrologists per million population is estimated to be 1.1 [57]. The lack of specialists must be addressed in order to have optimally functioning and widely accessible CKD facilities, as well as access to renal biopsy and renal replacement services. We must build on our current infrastructure.

Lastly, we need to empower our patients with knowledge regarding ART complications, and the monitoring and recognition of side effects. In over-burdened health facilities, it is not unusual for important management steps to be overlooked. Therefore, if we educate our patients, these can be avoided.

\section{Strengths and limitations}

An important strength of this study is the exhaustive search for relevant studies. Scoping review methodology is rigorous and methodical in its approach to examining the extent, range and nature of research activity in a particular field [18]. The unavoidable limitation of this study was the exclusion of literature published in other languages other than the English language.

\section{Conclusions}

The findings of the review are in keeping with that of international literature. South Africa has recently adopted WHO policy and as of September 2016, all HIV-infected patients have access to ART irrespective of CD4 cell count. Though this is a victory for the millions still awaiting ART in South Africa (in this context, particularly patients with undiagnosed HIVAN), the impact on the current health infrastructure that this strategy will have is unknown. More patients on ART equates to a further increase in chronic disease in South Africa, mainly CKD, hypertension and diabetes. With a lack of dedicated CKD clinics and specialist renal services particularly in the rural settings, as well as a deficiency in knowledge on the long-term impact of ART on CKD, this may be a victory for which the healthcare system could be ill prepared.

More research is urgently needed on the impact of ART on renal disease, ART-related renal complications and the prevalence of CKD, as well as cost effective methods for routine screening of renal disease in resource-poor settings.

\section{Additional files}

Additional file 1: Database Search Strategy. (DOCX $15 \mathrm{~kb}$ )

Additional file 2: PRISMA check list. (DOC $62 \mathrm{~kb}$ )

Additional file 3: Quality Appraisal tool. Two reviewers used the MMAT format to assess the quality of the content of the included studies. (DOCX $15 \mathrm{~kb}$ )

Additional file 4: Characteristics of studies included in the scoping review. (DOCX 26 kb) 


\section{Abbreviations}

ACR: Albumin creatinine ratio; AIDS: Acquired immunodeficiency syndrome; AKI: Acute kidney injury; ART: Antiretroviral treatment; CG: Cockcroft-Gault; Cl: Confidence interval; CKD: Chronic kidney disease; eGFR: Estimated glomerular filtration rate; ESRD: End stage renal disease; HIV: Human immunodeficiency virus; HIVAN: HIV associated nephropathy; ICGN: Immune complex glomerulonephritis; IDSA: Infectious Diseases Society of America; MCR: Microalbumin to creatinine ratio; MDRD: Modification of Diet in Renal Disease; MMAT: Mixed methods appraisal tool; PCR: Protein creatinine ratio; PRS: Protein reagent strip; TDF: Tenofovir disoproxil fumarate; UPCR: Urinary protein creatinine ratio; WHO: World Health Organization

\section{Acknowledgements}

We would like to thank the following institutions: College of Health Sciences and Library services at the University of KwaZulu-Natal for their support in providing us with resources to help with setting up and conducting this research study. The authors would also like to thank the librarians at Nelson R. Mandela Medical School (Mr. Msizi Khumalo and Mrs. Praba Naidoo) for the assistance with retrieving studies for the review. This study was funded by the College of Health Sciences research scholarship.

\section{Funding}

This study was funded by the University of KwaZulu-Natal, College of Health Sciences Research Scholarship.

\section{Availability of data and materials}

All data generated or analysed during this study are included in this published article and its Additional files files.

\section{Authors' contributions}

SA, NPM and TPM-T conceptualised and designed the study. SA prepared the first draft of the study and SMK contributed in the abstract, full article screening and the quality assessment of the included studies. TPMT contributed in the synthesis of data and the design of the sifting and data extraction processes. TPMT and NPM assisted with the manuscript preparation. All authors reviewed draft versions of the manuscript and approved the final version of the manuscript.

\section{Ethics approval and consent to participate}

Not applicable.

\section{Consent for publication}

Not applicable.

\section{Competing interests}

The authors declare that they have no competing interests.

\section{Publisher's Note}

Springer Nature remains neutral with regard to jurisdictional claims in published maps and institutional affiliations.

\section{Author details}

'Department of Internal Medicine, Nelson R. Mandela School of Medicine, University of KwaZulu-Natal, 719 Umbilo Road, Congella, Durban 4013, South Africa. ${ }^{2}$ Department of General Surgery, Nelson R. Mandela School of Medicine, University of KwaZulu-Natal, Durban, South Africa. ${ }^{3}$ Department of Public Health Medicine, School of Nursing and Public Health, University of KwaZulu-Natal, Durban, South Africa.

Received: 4 January 2017 Accepted: 6 October 2017

Published online: 13 October 2017

\section{References}

1. Boulle A, Bock P, Osler M, Cohen K, Channing L, Hilderbrand K, et al. Antiretroviral therapy and early mortality in South Africa. Bull World Health Organ. 2008;86(9):678-87.

2. National Antiretroviral treatment Guidelines, National Department of Health South Africa, First Edition, 2004. Available online at http://apps.who.int/ medicinedocs/documents/s17758en/s17758en.pdf

3. World Health Organisation. Guideline on when to start antiretroviral therapy and on pre-exposure prophylaxis for HIV Guidelines. World Health
Organisation: Geneva; September 2015. Available online at http://apps.who. int/iris/bitstream/10665/186275/1/9789241509565_eng.pdf.

4. Kalyesubula R, Wearne N, Semitala FC, Bowa K. HIV-associated renal and genitourinary comorbidities in Africa. J Acquir Immune Defic Syndr. 2014;67: S68-78.

5. Mayosi BM, Flisher AJ, Lalloo UG, Sitas F, Tollman SM, Bradshaw D. The burden of non-communicable diseases in South Africa. Lancet. 2009; 374(9693):934-47.

6. Msango L, Downs JA, Kalluvya SE, Kidenya BR, Kabangila R, Johnson WD Jr, et al. Renal dysfunction among HIV-infected patients starting antiretroviral therapy. AIDS (London, England). 2011;25(11):1421-5.

7. Bihl G. HIV-related renal disease-A clinical and practical approach in the South African context. South Afr J HIV Med. 2003;4(1):11-14.

8. Fabian J. Chronic kidney disease in HIV infection: early detec tion and preventive strategies. Contin Med Educ. 2007;28(8):372-6.

9. Stanifer JW, Jing B, Tolan S, Helmke N, Mukerjee R, Naicker S, et al. The epidemiology of chronic kidney disease in sub-Saharan Africa: a systematic review and meta-analysis. Lancet Glob Health. 2014;2(3):e174-e81.

10. Lucas GM, Ross MJ, Stock PG, Shlipak MG, Wyatt CM, Gupta SK, et al. Clinical practice guideline for the management of chronic kidney disease in patients infected with HIV: 2014 update by the HIV medicine association of the Infectious Diseases Society of America. Clin Infect Dis. 2014;59(9):e96-e138.

11. Fabian J, Katz I, Gerntholtz T, Goetsch S, Naicker S. Chronic kidney disease in human immunodeficiency virus infection. Panminerva Med. 2007;49(2):51-66.

12. Han T, Naicker S, Ramdial P, Assounga A. A cross-sectional study of HIVseropositive patients with varying degrees of proteinuria in South Africa. Kidney Int. 2006;69(12):2243-50.

13. D'Agati V, Appel GB. HIV infection and the kidney. J Am Soc Nephrol. 1997; 8(1):138-52.

14. Cohen SD, Kimmel PL. HIV-associated renal diseases in Africa-a desperate need for additional study. Nephrol Dial Transplant. 2007;22(8):2116-9.

15. Fabian J, Naicker S, Goetsch S, Venter WD. The clinical and histological response of HIV-associated kidney disease to antiretroviral therapy in South Africans. Nephrol Dial Transplant. 2013;28(6):1543-54.

16. Fabian J, Naicker S, Venter WD, Baker L, Naidoo S, Paget G, et al. Urinary screening abnormalities in antiretroviral-naive HIV-infected outpatients and implications for management-a single-center study in South Africa. Ethn Dis. 2009;19(1):80.

17. Gerntholtz TE, Goetsch SJW, Katz I. HIV-related nephropathy: a South African perspective. Kidney Int. 2006;69(10):1885-91.

18. Arksey H, O'Malley L. Scoping studies: towards a methodological framework. Int J Soc Res Methodol. 2005;8(1):19-32.

19. Pluye P, Robert E, Cargo M, Bartlett G, O'Cathain A, Griffiths F, Boardman F, Gagnon MP, Rousseau MC. Proposal: a mixed methods appraisal tool for systematic mixed studies. Available online at http:// mixedmethodsappraisaltoolpublic.pbworks.com/w/file/fetch/84371689/ MMAT\%202011\%20criteria\%20and\%20tutorial\%202011-06-29updated2014. 08.21.pdf.

20. Arendse C, Okpechi I, Swanepoel C. Acute dialysis in HIV-positive patients in Cape Town, South Africa. Nephrology. 2011;16(1):39-44.

21. Okpechi IG. Nephrotic syndrome in adult black South Africans: HIV-associated nephropathy as the main culprit. J Natl Med Assoc. 2010;102(12):1193.

22. Okpechi I, Swanepoel C, Duffield M, Mahala B, Wearne N, Alagbe S, et al. Patterns of renal disease in Cape Town South Africa: a 10-year review of a single-centre renal biopsy database. Nephrol Dial Transplant. 2010;26(6):1853-61.

23. van Rensburg BWJ, van Staden AM, Rossouw GJ, Joubert G. The profile of adult nephrology patients admitted to the Renal Unit of the Universitas Tertiary Hospital in Bloemfontein, South Africa from 1997 to 2006. Nephrol Dial Transplant. 2009;25(3):820-4.

24. Fabian J, Maher HA, Clark C, Naicker S, Becker P, Venter WD. Morbidity and mortality of black HIV-positive patients with end-stage kidney disease receiving chronic haemodialysis in South Africa. S Afr Med J. 2015;105(2):110-4.

25. Madala ND, Nkwanyana N, Dubula T, Naiker IP. Predictive performance of eGFR equations in south Africans of African and Indian ancestry compared with (9)(9)mTc-DTPA imaging. Int Urol Nephrol. 2012;44(3):847-55.

26. Kamkuemah M. Prevalence and incidence of renal dysfunction in patients initiating antiretroviral therapy at a primary health Care Centre in Gugulethu, cape town: a cohort study: University of Cape Town; 2013.

27. Brennan A, Evans D, Maskew M, Naicker S, Ive P, Sanne I, et al. Relationship between renal dysfunction, nephrotoxicity and death among HIV adults on tenofovir. AIDS (London, England). 2011;25(13):1603. 
28. Kasembeli AN, Duarte R, Ramsay M, Mosiane P, Dickens C, Dix-Peek T, et al. APOL1 risk variants are strongly associated with HIV-associated nephropathy in black South Africans. J Am Soc Nephrol. 2015;26(11):2882-90.

29. Vachiat Al, Musenge $\mathrm{E}$, Wadee $\mathrm{S}$, Naicker $\mathrm{S}$. Renal failure in HIV-positive patients_a South African experience. Clin Kidney J. 2013;6(6):584-9.

30. van Deventer HE, George JA, Paiker JE, Becker PJ, Katz IJ. Estimating glomerular filtration rate in black South Africans by use of the modification of diet in renal disease and Cockcroft-Gault equations. Clin Chem. 2008; 54(7):1197-202.

31. Wearne N, Swanepoel CR, Boulle A, Duffield MS, Rayner BL. The spectrum of renal histologies seen in HIV with outcomes, prognostic indicators and clinical correlations. Nephrol Dial Transplant. 2012;27(11):4109-18.

32. Kamkuemah M, Kaplan R, Bekker LG, Little F, Myer L. Renal impairment in HIV-infected patients initiating tenofovir-containing antiretroviral therapy regimens in a primary healthcare setting in South Africa. Trop Med Int Health. 2015;20(4):518-26.

33. Franey C, Knott D, Barnighausen T, Dedicoat M, Adam A, Lessells RJ, et al. Renal impairment in a rural African antiretroviral programme. BMC Infect Dis. 2009;9(1):143.

34. Madala ND, Thusi GP, Assounga AG, Naicker S. Characteristics of South African patients presenting with kidney disease in rural KwaZulu-Natal: a cross sectional study. BMC Nephrol. 2014;15(1):61.

35. Wensink GE, Schoffelen AF, Tempelman HA, Rookmaaker MB, Hoepelman AIM, Barth RE. Albuminuria is associated with traditional cardiovascular risk factors and viral load in HIV-infected patients in rural South Africa. PLoS One. 2015;10(8):1-14.

36. Genovese G, Friedman DJ, Ross MD, Lecordier L, Uzureau P, Freedman Bl, et al. Association of trypanolytic ApoL1 variants with kidney disease in African Americans. Science. 2010;329(5993):841-5.

37. The South African Antiretroviral Treatment Guidelines 2010, National department of Health South Africa, 2010. Available online at http://apps. who.int/medicinedocs/documents/s19153en/s19153en.pdf.

38. Herlitz LC, Mohan S, Stokes MB, Radhakrishnan J, D'Agati VD, Markowitz GS. Tenofovir nephrotoxicity: acute tubular necrosis with distinctive clinical, pathological, and mitochondrial abnormalities. Kidney Int. 2010;78(11):1171-7.

39. Cooper RD, Wiebe N, Smith N, Keiser P, Naicker S, Tonelli M. Systematic review and meta-analysis: renal safety of tenofovir disoproxil fumarate in HIV-infected patients. Clin Infect Dis. 2010;51(5):496-505.

40. Elias A, ljeoma O, Edikpo NJ, Oputiri D, Geoffrey O-BP. Tenofovir renal toxicity: evaluation of cohorts and clinical studies-part 2. Pharmacol Pharm. 2014;2014

41. Jafari A, Khalili H, Dashti-Khavidaki S. Tenofovir-induced nephrotoxicity: incidence, mechanism, risk factors, prognosis and proposed agents for prevention. Eur J Clin Pharmacol. 2014;70(9):1029-40.

42. Siedner MJ, Atta MG, Lucas GM, Perazella MA, Fine DM. Poor validity of urine dipstick as a screening tool for proteinuria in HIV-positive patients. J Acquir Immune Defic Syndr. 2008;47(2):261-3.

43. Szczech LA, Menezes P, Quinlivan EB, van der Horst C, Bartlett JA, Svetkey LP. Microalbuminuria predicts overt proteinuria among patients with HIV infection. HIV Medicine. 2010;11(7):419-26.

44. De León JIL, Mata-Marín JA, Andrade-Fuentes K, Huerta-Garcia G, Domínguez-Hemosillo JC, Gaytán-Martínez J. Strong correlation between protein reagent strip and protein-to-creatinine ratio for detection of renal dysfunction in HIV-infected patients: a cross-sectional study. AIDS Res Ther. 2015;12(1):1-5.

45. Stevens LA, Coresh J, Greene T, Levey AS. Assessing kidney function-measured and estimated glomerular filtration rate. N Engl J Med. 2006;354(23):2473-83.

46. Overton ET, Nurutdinova D, Freeman J, Seyfried W, Mondy KE. Factors associated with renal dysfunction within an urban HIV-infected cohort in the era of highly active antiretroviral therapy. HIV Med. 2009;10(6):343-50.

47. Wools-Kaloustian K, Gupta SK, Muloma E, Owino-Ong'or W, Sidle J, Aubrey RW, et al. Renal disease in an antiretroviral-naive HIV-infected outpatient population in Western Kenya. Nephrol Dial Transplant. 2007;22(8):2208-12.

48. Vali PS, Ismal K, Gowrishankar S, Sahay M. Renal disease in human immunodeficiency virus - not just HIV-associated nephropathy. Indian J Nephrol. 2012;22(2):98-102.

49. Lucas GM, Eustace JA, Sozio S, Mentari EK, Appiah KA, Moore RD. Highly active antiretroviral therapy and the incidence of HIV-1-associated nephropathy: a 12-year cohort study. AIDS. 2004;18(3):541-6.
50. Reid A, Stohr W, Walker S, Ssali F, Munderi P, Gilks C, editors. on behalf of the DART trial. Glomerular dysfunction and associated risk factors following initiation of ART in adults with HIV infection in Africa. Fifth IAS Conference on HIV Pathogenesis, Treatment and Prevention; 2005.

51. Scialla JJ, Atta MG, Fine DM. Relapse of HIV-associated nephropathy after discontinuing highly active antiretroviral therapy. AIDS. 2007;21(2):263-4.

52. Calza L, Trapani F, Tedeschi S, Piergentili B, Manfredi R, Colangeli V, et al. Tenofovir-induced renal toxicity in $324 \mathrm{HIV}$-infected, antiretroviral-naïve patients. Scand J Infect Dis. 2011;43(8):656-60.

53. Shisana O, Rehle T, Simbayi LC, Zuma K, Jooste S, Zungu N, Labadarios D, Onoya D, et al. South African national HIV prevalence, incidence and behaviour survey, 2012. Cape Town: HSRC Press; 2014. p. 195.

54. Wensink GE, Schoffelen AF, Tempelman HA, Rookmaaker MB, Hoepelman Al, Barth RE. Albuminuria is associated with traditional cardiovascular risk factors and viral load in HIV-infected patients in rural South Africa. PLoS One. 2015;10(8):e0136529.

55. Longo AL, Lepira FB, Sumaili EK, Makulo JRR, Mukumbi H, Bukabau JB, et al. Prevalence of low estimated glomerular filtration rate, proteinuria, and associated risk factors among HIV-infected black patients using CockroftGault and modification of diet in renal disease study equations. J Acquir Immune Defic Syndr. 2012;59(1):59-64.

56. Ando M, Yanagisawa N, Ajisawa A, Tsuchiya K, Nitta K. A simple model for predicting incidence of chronic kidney disease in HIV-infected patients. Clin Exp Nephrol. 2011;15(2):242-7.

57. Katz IJ, Gerntholtz T, Naicker S. Africa and nephrology: the forgotten continent. Nephron Clin Pract. 2011;117(4):320-7.

\section{Submit your next manuscript to BioMed Central and we will help you at every step:}

- We accept pre-submission inquiries

- Our selector tool helps you to find the most relevant journal

- We provide round the clock customer support

- Convenient online submission

- Thorough peer review

- Inclusion in PubMed and all major indexing services

- Maximum visibility for your research

Submit your manuscript at www.biomedcentral.com/submit
C) Biomed Central 